\title{
Fortgeschrittenes Nierenzellkarzinom
}

\section{Lenvatinib in Kombination mit Everolimus als Zweitlinientherapie zugelassen}

Seit August 2016 ist Lenvatinib (Kisplyx ${ }^{\circledR}$ ) in Kombination mit Everolimus (Afinitor ${ }^{\circledR}$ ) zur Behandlung von erwachsenen Patienten mit fortgeschrittenem Nierenzellkarzinom (RCC) nach einer vorherigen, gegen den vaskulären endothelialen Wachstumsfaktor (VEGF) gerichteten Therapie zugelassen. Durch die Zugabe des Multikinase-Inhibitors zur Standard-Zweitlinientherapie mit Everolimus konnte das progressionsfreie Überleben (primärer Endpunkt) im Vergleich mit Everolimus signifikant von 5,5 auf 14,6 Monate verlängert werden. Das Gesamtüberleben verlängerte sich durch die Zugabe von Lenvatinib um 10,1 Monate auf 25,5 Monate. Es handelt sich um die erste Zulassung einer Kombination von zwei zielgerichteten Substanzen beim RCC.

Einer Schätzung des Robert Koch-Instituts zufolge werden im Jahr 2016 in Deutschland 16500 Menschen an einem malignen Tumor der Niere erkranken. Etwa 90\% davon sind Nierenzellkarzinome [1]. Zum Diagnosezeitpunkt haben $25-30 \%$ der Tumore bereits Metastasen gebildet. Die relative 5-Jahres-Überlebensrate liegt im Stadium IV bei $14 \%$. In der palliativen Situation kommen zielgerichtete Wirkstoffe zum Einsatz. Nach Versagen einer gegen den VEGF gerichteten Therapie steht der mTOR-Inhibitor Everolimus zur Verfügung, nach Sunitinib kann auch Axitinib ge- geben werden [2]. Mit der neuen Option Lenvatinib plus Everolimus wurde erstmals eine Kombination von zwei zielgerichteten Substanzen beim RCC zugelassen. Aufgrund der überzeugenden Daten erfolgte die Zulassung durch die Europäische ArzneimittelAgentur (EMA) in einem beschleunigten Verfahren.

\section{Design der Zulassungsstudie}

Basis für die Zulassung waren die Ergebnisse der globalen, multizentrischen, offenen PhaseII-Studie 205, in der Lenvatinib in Kombination mit Everolimus jeweils mit Lenvatinib* und Everolimus als Monotherapie verglichen wurde. Eingeschlossen waren Patienten mit einem klarzelligen, lokal fortgeschrittenen oder metastasierten Nierenzellkarzinom und gutem Allgemeinzustand (ECOG-Performance-Status 0 oder 1), deren Erkrankung unter oder innerhalb von 9 Monaten nach einer antiangiogenetischen Erstlinientherapie fortgeschritten war [3]. Die Teilnehmer erhielten randomisiert entweder Lenvatinib (18 mg/Tag) in Kombination mit Everolimus ( $5 \mathrm{mg} / \mathrm{Tag}$ ) oder Lenvatinib* (24 mg/Tag) oder Everolimus (10 $\mathrm{mg} / \mathrm{Tag})$. Alle Wirkstoffe wurden oral verabreicht. Die Therapie erfolgte in kontinuierlichen Zyklen von 28 Tagen und wurde fortgeführt bis zum Krankheitsprogress oder inakzeptabler Toxizität. Primärer Endpunkt war das unabhängig erhobene progressionsfreie

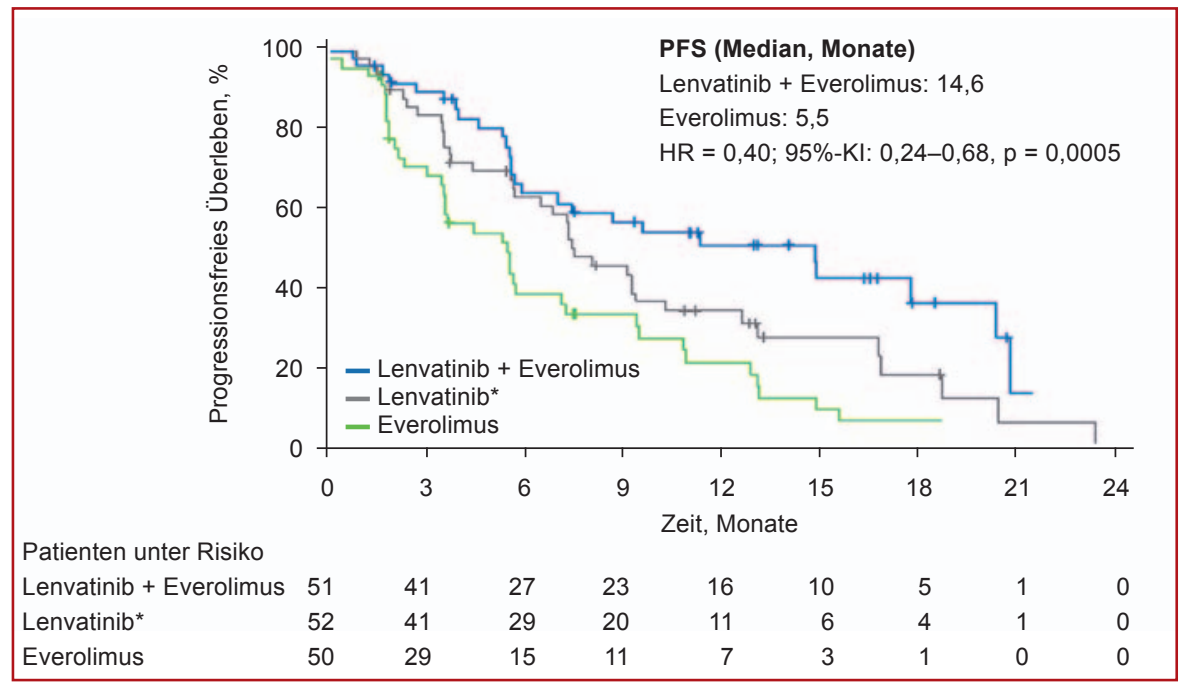

Abb. 1. Progressionsfreies Überleben in der für die Zulassung von Lenvatinib in Kombination mit Everolimus maßgeblichen Studie 205; modifiziert nach [3].
Überleben (PFS), definiert als Zeitspanne von der Randomisierung bis zur Krankheitsprogression oder Tod. Sekundäre Endpunkte beinhalteten Gesamtansprechrate (ORR), Gesamtüberleben und Verträglichkeit [3].

\section{Alle Patienten mit metastasierter Erkrankung}

Insgesamt 153 Patienten wurden eingeschlossen und im Verhältnis 1:1:1 auf die 3 Therapiearme randomisiert. Im Median waren die Teilnehmer 61 Jahre alt und bei etwa zwei Dritteln (61\%) lag nach Heng ein intermediäres Progressionsrisiko vor. Alle Teilnehmer hatten Metastasen, bei der Hälfte von ihnen waren es mehr als drei. Am häufigsten fanden sich Metastasen in Lunge und Lymphknoten. Überwiegend (65\%) hatten die Patienten eine Vortherapie mit Sunitinib erhalten [3].

\section{Nahezu Verdreifachung des PFS gegenüber Everolimus}

Bei Patienten, die mit der Kombinationstherapie behandelt wurden $(n=51)$, betrug das PFS im Median 14,6 Monate. Im Vergleich dazu waren Patienten in der Everolimus-Gruppe $(\mathrm{n}=50)$ 5,5 Monate progressionsfrei (Hazard Ratio $(\mathrm{HR})=0,40 ; 95 \%$-Konfidenzintervall (KI): 0,24-0,68; p = 0,0005; Abb. 1). Die Kombination von Lenvatinib plus Everolimus führte somit zu einer Reduktion des Progressionsrisikos von $60 \%$ gegenüber Everolimus allein. Nach einem Jahr waren 51\% der Patienten im Kombinationsarm und 21\% der EverolimusPatienten noch ohne Progress [3].

«Die Daten der Zulassungsstudie zeigen, dass die zusätzliche Gabe von Lenvatinib im Vergleich zu einer Everolimus-Monotherapie bei Patienten mit einem fortgeschrittenen oder metastasierten Nierenzellkarzinom zu einer statistisch signifikanten Verbesserung des progressionsfreien Überlebens führt. Dadurch haben sie die Chance, länger ohne ein Fortschreiten ihrer Erkrankung zu leben. Das sind ermutigende Nachrichten sowohl für uns als behandelnde Ärzte als auch für die betroffenen Patienten, die an einer schweren Krebserkrankung leiden», so Prof. Axel Merseburger, Lübeck.

*Die Lenvatinib Monotherapie ist nicht für die Behandlung des fortgeschrittenen Nierenzellkarzinoms zugelassen. 


\section{Klinisch relevante Ansprechrate} von $43 \%$

Auch beim Ansprechen zeigte sich ein klarer Vorteil für die Kombinationstherapie. Die Gesamtansprechrate betrug $43 \%$ bei Patienten im Kombinationsarm (2\% komplette und 41\% partielle Remissionen) im Vergleich mit 6\% (ausschließlich partielle Remissionen) im Everolimus-Arm (Risk-Ratio $=7,2 ; 95 \%-\mathrm{KI}$ : 2,3-22,5; $\mathrm{p}<0,0001)$. Im Median sprachen die Patienten 13 Monate auf die Therapie mit Lenvatinib in Kombination mit Everolimus und 8,5 Monate auf Everolimus allein an [3].

\section{Gesamtüberleben von über 2 Jahren}

Patienten, welche die Kombinationstherapie Lenvatinib plus Everolimus erhielten, lebten im Median 10,1 Monate länger als mit der Everolimus-Monotherapie. Das Gesamtüberleben betrug im Kombinationsarm 25,5 Monate gegenüber 15,4 Monaten unter Everolimus (HR $=0,51 ; 95 \%-\mathrm{KI}: 0,30-0,88 ; \mathrm{p}=0,024$ - Datenschnitt Dezember 2014). Zum Zeitpunkt der primären Analyse betrug das mediane Gesamtüberleben 25,5 Monate für die Kombination versus 17,5 Monate für Everolimus allein (HR $=0,55 ; 95 \%-\mathrm{KI}: 0,30-1,01 ; \mathrm{p}=$ 0,062 - Datenschnitt Juni 2014). Nach einem Jahr lebten noch 75\% der Patienten im Kombinations-Arm und $62 \%$ in der EverolimusGruppe, nach 18 Monaten waren es $65 \%$ bzw. 41\% [3]. Ein bei der Jahrestagung der Amerikanischen Krebsgesellschaft (ASCO) vorgestelltes Update ergab ein Gesamtüberleben von 25,5 Monaten für Lenvatinib in Kombination mit Everolimus versus 15,4 Monate für Everolimus allein ( $\mathrm{HR}=0,59$; 95\%-KI: 0,36-0,97; $\mathrm{p}=0,065$ - Datenschnitt Juli 2015) [4].

«Mit Lenvatinib plus Everolimus haben wir zum ersten Mal eine Kombination von zwei zielgerichteten Wirkstoffen, die beim metastasierten Nierenzellkarzinom eine synergistische Wirksamkeit gezeigt hat. Durch die Kombination des Tyrosinkinase-Inhibitors Lenvatinib mit dem mTOR-Inhibitor Everolimus werden wesentliche Zielstrukturen beim fortgeschrittenen Nierenzellkarzinom gleichzeitig adressiert. Mit der gezeigten Effektivität bezüglich des PFS, des Ansprechens und des Gesamtüberlebens stellt die Kombination von Lenvatinib plus Everolimus eine weitere Therapieoption in der Zweitlinientherapie dar», betont Prof. Jürgen Gschwend, München.

\section{Aufnahme in die NCCN- Guidelines}

Die Kombination von Lenvatinib plus Everolimus wurde bereits in die Nierenkrebs-Guideline des US-amerikanischen National Comprehen- sive Cancer Network (NCCN) aufgenommen. Sie wird mit dem zweithöchsten Empfehlungsgrad (2a) zur Therapie bei RCC-Patienten empfohlen, die zuvor eine antiangiogenetische Behandlung erhalten haben [5].

\section{Verträglichkeitsprofil konsistent mit Einzelsubstanzen}

Die häufigsten therapiebedingten unerwünschten Ereignisse aller Grade $(>30 \%)$ in der Zulassungsstudie waren unter Lenvatinib plus Everolimus Diarrhoe (85\%), Fatigue bzw. Asthenie (59\%), verminderter Appetit (51\%), Erbrechen (45\%), Bluthochdruck (41\%), Übelkeit (41\%), Husten (37\%), Hypercholesterolämie (33\%), Hypertriglyzeridämie (35\%) und Gewichtsverlust (31\%) [3]. Unerwünschte Ereignisse von Grad 3 oder 4 traten bei $71 \%$ der Patienten im Kombinationsarm und bei $50 \%$ im Everolimus-Arm auf. Zu den häufigsten Nebenwirkungen von Grad 3 unter Lenvatinib plus Everolimus gehörten Diarrhoe (20\%), Fatigue oder Asthenie und Bluthochdruck (jew. 14\%). Unter der EverolimusMonotherapie waren dies Anämie, Dyspnoe, Hypertriglyzeridämie und Hyperglykämie [3]. Schwerwiegende unerwünschte Ereignisse vom Grad $\geq 3$ traten bei $45 \%$ der Patienten mit Lenvatinib plus Everolimus auf und bei $38 \%$ im Everolimus-Arm [3]. Bei 71\% bzw. 2\% der Patienten im Kombinationsarm erfolgte eine Dosisreduktion von Lenvatinib bzw. Everolimus; meist wurde die Dosisreduktion von Lenvatinib in den ersten 3 Therapiezyklen vorgenommen. 24\% der Patienten im Kombinationsarm und $12 \%$ im Everolimus-Arm brachen die Therapie aufgrund unerwünschter Ereignisse ab [3].

\section{Therapiemanagement in} Therapieplanung integrieren

«Das Verträglichkeitsprofil der Kombination in der Zulassungsstudie war konsistent mit den bekannten Nebenwirkungen der Einzelsubstanzen. Aufgrund langjähriger Erfahrungen mit dem Einsatz von antiangiogenetisch wirkenden Tyrosinkinase-Hemmern und mTOR-Inhibitoren sind die meisten Onkologen mit den typischen Nebenwirkungen dieser Substanzklassen vertraut. Das sind gute Voraussetzungen für die Handhabbarkeit der Kombinationstherapie in der klinischen Praxis», so die Einschätzung von Prof. Merseburger. Insbesondere zu Beginn der Therapie empfiehlt sich ein engmaschiges Monitoring. Ein proaktives Therapiemanagement umfasst in Hinblick auf potenzielle Nebenwirkungen wie Übelkeit, Erbrechen, Diarrhoe und Bluthochdruck geeignete prophylaktische und begleitende Interventionen. In schweren Fällen kann die Dosis von Lenvatinib reduziert oder die Therapie unterbrochen werden.
Spezifischer Wirkmechanismus Lenvatinib ist ein Multityrosinkinase-Inhibitor, der gleichzeitig die Aktivität des VEGFR, des Fibroblastenwachstumsfaktor-Rezeptors (FGFR), von RET, KIT und des thrombozytären Wachstumsfaktor-Rezeptors (PDGFR) inhibiert $[6,7]$. Darüber hinaus erfolgt die Bindung von Lenvatinib bei der Kinase-Inhibition über einen neuen, sogenannten Typ-VBindungsmodus, der durch eine schnelle und langanhaltende Bindung charakterisiert ist. Dieser unterscheidet sich vom Bindungsmodus anderer bekannter TKI, bei denen es sich überwiegend um Typ-I- oder Typ-II-Inhibitoren handelt [8].

\section{Fazit}

Die Kombination Lenvatinib plus Everolimus führte in der Zulassungsstudie im Vergleich zu Everolimus zu einem signifikant verlängerten PFS. Darüber hinaus konnte das Gesamtüberleben um 10 Monate auf über 2 Jahre verlängert werden. Das Nebenwirkungsprofil war konsistent mit dem der jeweiligen Einzelsubstanzen. Die unter Lenvatinib beobachteten klassenspezifischen Nebenwirkungen einer gegen den VEGF gerichteten Therapie sind mit einem geeigneten Therapiemanagement handhabbar. Die Kombination hat das Potenzial für einen neuen Standard in der Zweitlinientherapie des fortgeschrittenen oder metastasierten RCC.

Anne Krampe-Scheidler, Berlin

\section{Literatur}

1 Krebs in Deutschland 2011/2012. 10. Ausgabe. Berlin, 2015.

2 Diagnostik, Therapie und Nachsorge des Nierenzellkarzinoms, Langversion 1.0, 2015; http://leitlinienprogramm-onkologie.de/Leitlinien.7.0.html (Zugriff am 18.07.2016).

3 Motzer R et al.: Lancet Oncol 2015;16:1473-1482.

4 Hutson TE et al.: J Clin Oncol 2016;34(suppl; abstr 4553); poster \#175.

5 NCCN Clinical Practice Guidelines in Oncology (NCCN Guidelines ${ }^{\circledR}$ ): Kidney Cancer, Version 3.2016; https://www.nccn.org/professionals/physician_ gls/f_guidelines.asp\#site (Zugriff am 20.7.2016).

6 Matsui J et al.: Clin Cancer Res 2008;14:5459-5465.

Matsui J et al.: Int J Cancer 2008;122:664-671.

8 Okamoto K et al.: ACS Med Chem Lett 2015;6:89-94.

Impressum

Fortgeschrittenes Nierenzellkarzinom

Lenvatinib in Kombination mit Everolimus als Zweitlinientherapie zugelassen

PharmaForum in

OnCology ReseARCH AND TREATMENT 39 | 10 | 16

(C) 2016 by S. Karger Verlag für Medizin

und Naturwissenschaften $\mathrm{GmbH}$

Wilhelmstraße 20A

79098 Freiburg, Deutschland

Mit freundlicher Unterstützung durch

Eisai $\mathbf{G m b H}$

Verlag, Herausgeber, Redaktion und Verlagsgeschäftsführung übernehmen keine Verantwortung für den Inhalt dieser Rubrik. 